\title{
Assessment of coronary reperfusion in patients with myocardial infarction using fatty acid binding protein concentrations in plasma
}

\author{
M J M de Groot, A M M Muijtjens, M L Simoons, W T Hermens, J F C Glatz
}

\begin{abstract}
Objective-To examine whether successful coronary reperfusion after thrombolytic treatment in patients with confirmed acute myocardial infarction can be diagnosed from the plasma marker fatty acid binding protein (FABP), for either acute clinical decision making or retrospective purposes.

Design-Retrospective substudy of the GUSTO trial.

Setting-10 hospitals in four European countries.

Patients - 115 patients were treated with thrombolytic agents within six hours after the onset of acute myocardial infarction. Patency of the infarct related artery was determined by angiography within 120 minutes of the start of thrombolysis.

Main outcome measures-First hour rate of increase in plasma FABP concentration after thrombolytic treatment, compared with increase in plasma myoglobin concentration and creatine kinase isoenzyme $\mathrm{MB}(\mathrm{CK}-\mathrm{MB})$ activity. Infarct size was estimated from the cumulative release of the enzyme $\alpha$ hydroxybutyrate dehydrogenase in plasma during 72 hours, or from the sum of ST segment elevations on admission. Logistic regression analyses were performed to construct predictive models for patency.

Results-Complete reperfusion (TIMI 3) occurred in 50 patients, partial reperfusion (TIMI 2) in 36, and no reperfusion (TIMI 0+1) in 29. Receiver operating characteristic (ROC) curve analyses showed that the best performance of FABP was obtained when TIMI scores 2 and 3 were grouped and compared with TIMI score $0+1$. The performance of FABP as a reperfusion marker was improved by combining it with $\alpha$ hydroxybutyrate dehydrogenase infarct size, but not with an early surrogate of infarct size (ST segment elevation on admission). In combination with infarct size FABP performed as well as myoglobin (areas under the ROC curve 0.868 and 0.857 , respectively) and better than $\mathrm{CK}-\mathrm{MB}$ (area $=0.796$ ). At optimum cut off levels, positive predictive values were $97 \%$ for FABP, 95\% for myoglobin, and $89 \%$ for CK-MB (without infarct size, $87 \%, 88 \%$, and $87 \%$, respectively), and negative predictive values were $55 \%, 52 \%$, and $50 \%$, respectively (without infarct size, $44 \%, 42 \%$, and $34 \%$ ).

Conclusions-FABP and myoglobin perform equally well as reperfusion markers, and successful reperfusion can be assessed, with positive predictive values of $87 \%$ and $88 \%$, or even $97 \%$ and $95 \%$ when infarct size is also taken into account. However, identification of non-reperfused patients remains a problem, as negative predictive values will generally remain below $70 \%$.

(Heart 2001;85:278-285)
\end{abstract}

Keywords: myocardial reperfusion; cardiac marker proteins

Cardiovascular

Research Institute

Maastricht (CARIM),

Maastricht University

PO Box 616, 6200 MD

Maastricht,

Netherlands

M J M de Groot

W T Hermens

J F C Glatz

Department of

Educational

Development and

Research, Maastricht

University

A M M Muijtjens

Thoraxcenter, Erasmus University

Rotterdam,

Netherlands

M L Simoons

Correspondence to: Dr Glatz

glatz@fys.unimaas.nl

Accepted 28 September 2000
Thrombolytic treatment has become important in patients with acute myocardial infarction in order to reopen the infarct related artery and improve the survival of heart muscle. However, when this treatment has failed the patient may need other interventions such as angioplasty. In order to reach a decision on this, it is important to make a rapid, accurate, and non-invasive evaluation of the reperfusion status of the infarct related coronary artery after initial thrombolytic treatment. Relief of chest pain, normalisation of ST segment elevation on the ECG, and the occurrence of specific arrhythmias have been used as markers of reperfusion, but their sensitivity and specificity are limited. ${ }^{12}$ In addition, early washout and peaking of various cardiac proteins such as creatine kinase, creatine kinase $\mathrm{MB}$ isoenzyme (CK-MB), and myoglobin have been used to identify coronary reperfusion..$^{3-6}$ Of these biochemical markers, myoglobin has been found most valuable because of its rapid release after opening of the vessel. ${ }^{367}$ More recently, a new plasma marker - fatty acid binding protein (FABP) - has become available, which resembles myoglobin in size $(15 \mathrm{kDa})$, in its abundant presence in the cytoplasm of cardiomyocytes, and in its similarly rapid release into plasma after reperfusion. ${ }^{8} 9$ FABP has been found superior to myoglobin for the early detection of acute myocardial infarction. ${ }^{11}{ }^{11}$

It was our aim in the present study to evaluate whether FABP can be used as a marker, either in acute clinical situations or retrospectively, to distinguish successful reperfusion from persistent occlusion after thrombolytic treatment in patients with confirmed acute myocardial infarction. We determined the increase in plasma concentrations of FABP in the first hour after thrombolytic treatment, and we also examined the increases occurring from 1-3 hours after thrombolysis as well as certain clinical variables (treatment delay, delay in performing angiography, infarct location). A comparison was made with the established markers myoglobin and CK-MB. The increase in 
cardiac proteins in the plasma after infarction is dependent on infarct size. We therefore assessed infarct size by an established methodthe cumulative release of $\alpha$ hydroxybutyrate dehydrogenase during the first 72 hours after infarction. ${ }^{12}$ However, only a retrospective assessment of reperfusion status can be obtained in this way, though acute assessment is clinically more relevant. We therefore attempted to use an early surrogate for infarct size - initial ST segment elevation on the ECG.

\section{Methods}

PATIENTS

Data were obtained from 124 patients with confirmed acute myocardial infarction, enrolled in the GUSTO (global utilisation of streptokinase and tissue plasminogen activator for occluded coronary arteries) enzyme substudy. ${ }^{13}$ Inclusion criteria for enrolment into the GUSTO study have been described in detail elsewhere. ${ }^{14}$ In summary, patients were eligible when they were admitted to hospital within six hours after the onset of symptoms, had chest pain lasting for at least 20 minutes, and showed ECG evidence of acute myocardial infarction $(\geqslant 0.1 \mathrm{mV}$ of $\mathrm{ST}$ segment elevation in two or more limb leads, or $\geqslant 0.2 \mathrm{mV}$ in two or more contiguous precordial leads). Patients suspected of acute myocardial infarction received one of four intravenous thrombolytic regimens: streptokinase with subcutaneous heparin; streptokinase with intravenous heparin; accelerated tissue plasminogen activator (t-PA) with intravenous heparin; or the combination of t-PA and streptokinase, along with intravenous heparin.

Following thrombolytic treatment, coronary angiography was performed within 120 minutes (mean (SEM) $1.6(0.2)$ hours, range 0.92.0 hours) to determine the reperfusion status of the infarct related artery. Flow in the infarct related artery was graded according to the thrombolysis in myocardial infarction (TIMI) trial classification. ${ }^{15}$ Coronary arteries with TIMI grade 3 flow were regarded as successfully reperfused. Coronary arteries with TIMI grade 2 flow were regarded as partially open. TIMI grade 0 or 1 defined persistent occlusion.

\section{BLOOD SAMPLING}

Blood samples were collected immediately before and at 1, 3, 6, 12, 18, 24, 36, 48, 72, and 96 hours after the start of thrombolytic treatment, resulting in 11 samples/patient. Exact sampling time was recorded on the GUSTO enzyme case report form. ${ }^{13}$ Samples were collected in glass tubes containing dry heparin to prevent clotting. After routine centrifugation, plasma was kept at $-20^{\circ} \mathrm{C}$ in the local hospital and within eight weeks was transported in polystyrene boxes with dry ice to the central laboratory at Maastricht, Netherlands, and stored at $-80^{\circ} \mathrm{C}$ until assays were performed.

ANALYTICAL TECHNIQUES

Heart type FABP was measured in duplicate in plasma samples by a non-competitive enzyme linked immunosorbent assay (ELISA) as described elsewhere ${ }^{16}$ using an incubation time of 60 minutes. Samples were diluted with phosphate buffered saline $(\mathrm{pH}$ 7.4) containing $0.1 \%$ bovine serum albumin and $0.05 \%$ Tween-20. The detection limit of the assay was $0.2 \mu \mathrm{g} / \mathrm{l}$. Quality control was performed with human plasma, spiked with recombinant human FABP. Intra-assay and interassay imprecision values were $4.2 \%$ and $9.0 \%$, respectively. Myoglobin was determined in duplicate by a turbidimetric immunoassay (Unimate 3 Myo, art 0751839, Roche, Mijdrecht, Netherlands) on a Cobas Fara analyser (Roche Diagnostic Systems, Basel, Switzerland). Plasma samples were diluted with saline $(0.9 \% \mathrm{NaCl})$. For quality control a commercial standard was used (Roche, art 075186). Intra-assay and interassay imprecision values were $3.5 \%$ and $4.0 \%$, respectively. It has been reported in detail previously that FABP and myoglobin concentrations in plasma stored at $-80^{\circ} \mathrm{C}$, as in the present study, remain stable for several years. ${ }^{17}$ Plasma concentrations of FABP and myoglobin are expressed in $\mu \mathrm{g} / \mathrm{l}$.

Activities of CK-MB and $\alpha$ hydroxybutyrate dehydrogenase were measured spectrophotometrically in duplicate at $25^{\circ} \mathrm{C}$, using a centrifugal analyser (Cobas Bio System, Roche) and commercially available test kits. The CK-MB test is based on immunoinhibition of the predominant $\mathrm{M}$ unit in creatine kinase (Boehringer Mannheim, Mannheim, Germany). The $\alpha$ hydroxybutyrate dehydrogenase test is based on the preferential catalytic activity of the myocardial isoforms $\mathrm{LDH}_{1}$ and $\mathrm{LDH}_{2}$ of lactate dehydrogenase in the conversion of $\alpha$ ketobutyrate, instead of pyruvate (Diagnostica Merck, Darmstadt, Germany). Quality control was performed using a commercially available control serum (Precipath, Boehringer Mannheim). Intra-assay and interassay imprecision values were $3.2 \%$ and $6.6 \%$ for CK-MB and $2.4 \%$ and $4.4 \%$ for $\alpha$ hydroxybutyrate dehydrogenase. Activities are expressed in micromoles of substrate converted per minute and litre of plasma (U/1).

CALCULATION OF THE INCREASE RATE OF PLASMA FABP, MYOGLOBIN, AND CK-MB

The first hour increases in plasma FABP, myoglobin, and CK-MB release were calculated by the difference in plasma concentrations or activity between the first two samples - that is, the samples just before $(-0.11(0.02)$ hours $)$ and about one hour $(1.14$ (0.03)) after thrombolytic treatment, divided by the exact time between these two samples. The increase in plasma FABP, myoglobin, and CK-MB release was also determined from 1-3 hours $(2.97(0.02))$ after thrombolytic treatment. The increase rates are expressed in $\mu \mathrm{g} / \mathrm{l} /$ hour for FABP and myoglobin, and in $\mathrm{U} / \mathrm{l} /$ hour for CK-MB.

DETERMINATION OF ENZYMATIC INFARCT SIZE Infarct size was calculated from the cumulative release of $\alpha$ hydroxybutyrate dehydrogenase activity during 72 hours. Cumulative release of $\alpha$ hydroxybutyrate dehydrogenase per litre of 
plasma, from the onset of acute myocardial infarction $(\mathrm{t}=0)$ up to time $\mathrm{t}$, is indicated as $\mathrm{Q}(\mathrm{t})$ and was calculated from the following expres$\operatorname{sion}^{12}$ :

$$
\begin{aligned}
& Q(t)= C(t)+\operatorname{TER} \int_{0}^{t} C(\tau) \exp [\operatorname{ERR}(\tau-t)] \\
& \mathrm{d} \tau+\mathrm{FCR} \int_{0}^{t} C(\tau) \mathrm{d} \tau
\end{aligned}
$$

The three terms are the quantity of released protein still present in plasma at time $t$, the extravasated quantity of protein at time $t$, and the quantity of protein eliminated from plasma up to time $t$, all three being expressed per litre of plasma.

$\mathrm{C}(\mathrm{t})$ is the plasma protein concentration or enzyme activity at time $t$, corrected by subtraction of normal steady state values, Cs. The latter were obtained from the lowest plasma values if they did not exceed the maximum value of $120 \mathrm{U} / 1$ for $\alpha$ hydroxybutyrate dehydrogenase. Otherwise, fixed mean values of $82 \mathrm{U} / 1$ were used. The variables TER and ERR represent the fractional rate constants for transcapillary escape and extravascular return of protein. Parameter values for $\alpha$ hydroxybutyrate dehydrogenase in man are TER $=0.014 / \mathrm{h}$ and $\mathrm{ERR}=0.018 / \mathrm{h} .{ }^{12} \mathrm{FCR}$ is the fractional catabolic rate constant for the elimination of protein from plasma $(0.015 / \mathrm{h})$. Infarct size is expressed in gram equivalents of myocardium/ litre of plasma (g eq/1). To this end, cumulative release of $\alpha$ hydroxybutyrate dehydrogenase/ litre of plasma was divided by the mean myocardial content of $\alpha$ hydroxybutyrate dehydrogenase/g wet weight of tissue $(123 \mathrm{U} / \mathrm{g})$.

\section{ST SEGMENT ELEVATION}

The sum of ST segment elevations was obtained from admission ECG recordings. ST segment elevation and depression were measured at the J point of the ECG. The degree of elevation or depression was measured in millivolts. Total ST segment elevations were calculated as described elsewhere. ${ }^{18}$

DATA ANALYSIS

Data analysis was performed with standard software (SPSS). Mean values (SEM) were calculated. Differences between the rates of increase in the TIMI groups were tested by the non-parametric Mann-Whitney rank test.

Predictive models for patency were constructed using logistic multiple regression. This method can be used when a binary classification, such as reperfusion versus nonreperfusion, is explained or predicted by a set of continuous variables ${ }^{19}$ (for example, first hour increase rate of a plasma marker concentration, infarct size, and so on). In the corresponding model the log odds of the probability of reperfusion (q) was expressed as a linear function of the explaining variables: $\log (\mathrm{q} /(1-\mathrm{q}))=\mathrm{b} 0+\mathrm{b} 1 \times($ first hour increase of $\mathrm{FABP})+\mathrm{b} 2 \times($ infarct size $)$.

Variables that contributed significantly to correct prediction of the classification were identified in a stepwise logistic regression pro- cedure. Estimates of the variables b0, b1, . . were obtained and for each case the estimated probability of reperfusion, q, was calculated. In our approach, q was used as an indicator of reperfusion analogous to the outcome in a diagnostic test. High values of $\mathrm{q}$ indicated reperfusion, low values indicated that reperfusion was not obtained. In order to distinguish high and low values, a cut off for $\mathrm{q}$ is required. In a receiver operating characteristic (ROC) curve, analysis of the relation between $\mathrm{q}$ and reperfusion, indicated by the TIMI score (true classification), was investigated for varying levels of cut off. The predictive value of a combination of predictors could thus be evaluated in a standard ROC analysis.

The sensitivity, specificity, positive predictive value (PPV), and negative predictive value (NPV) were determined as follows:

Sensitivity - percentage of correctly identified patients with patent (TIMI 2 or 3 ) infarct related artery;

Specificity-percentage of correctly identified patients with occluded (TIMI 0 or 1 ) infarct related artery;

$P P V$ - percentage of correctly identified patients with reperfusion;

$N P V$ - percentage of correctly identified patients with occlusion.

Plots were made of the sensitivity (true positive rate) against 1 - specificity (false positive rate) (ROC curve), and statistical comparison of the areas under the curve was performed according to a previously described method. ${ }^{20}$ Relations between variables were calculated by Pearson's correlation coefficients. Probability values of $\mathrm{p}<0.05$ were considered significant.

\section{Results}

PATIENTS

Patients who had cardioversion $(\mathrm{n}=9)$ were excluded from the present study because of possible skeletal muscle damage. Baseline characteristics of the remaining 115 patients - 93 men and 22 women - are shown in table 1. Except for the percentage of women, there were no significant differences in baseline characteristics, so for further analysis data from the four treatment groups were taken together. Fifty patients $(43 \%)$ obtained a TIMI 3 score after angiography, 36 patients $(31 \%)$ obtained a TIMI 2 score, while the other 29 patients $(25 \%)$ had a score of TIMI 0 or 1 . For the 115 patients studied, all variables that might be associated with reperfusion were complete.

PREDICTION OF REPERFUSION CONSIDERING TIMI 3 SCORE SEPARATELY OR COMBINED WITH TIMI SCORE 2

The concentrations of FABP, myoglobin, and CK-MB in plasma samples obtained immediately before thrombolytic treatment did not differ significantly among the TIMI score groups (data not shown). One hour after thrombolytic treatment, plasma FABP, myoglobin, and CK-MB concentrations had increased significantly more in the TIMI 2 and 3 patients $(\mathrm{p}<0.005$ for FABP and myoglobin, $p<0.05$ for $C K-M B)$ than in the TIMI 0 or TIMI 1 patients, but for each 
Table 1 Baseline characteristics of the different treatment groups

\begin{tabular}{|c|c|c|c|c|c|}
\hline & All patients & $\begin{array}{l}\text { SK with sc } \\
\text { heparin }\end{array}$ & $\begin{array}{l}\text { SK with iv } \\
\text { heparin }\end{array}$ & $\begin{array}{l}\text { tPA with iv } \\
\text { heparin }\end{array}$ & $\begin{array}{l}S K+t P A \\
\text { with iv } \\
\text { heparin }\end{array}$ \\
\hline \multicolumn{6}{|l|}{ Baseline variables } \\
\hline Number of patients & 115 & 32 & 18 & 39 & 26 \\
\hline Age (years) & $59(1)$ & $61(2)$ & $62(2)$ & $58(2)$ & $57(2)$ \\
\hline Female sex (\%) & 19 & 19 & 39 & 21 & 4 \\
\hline \multicolumn{6}{|l|}{ Infarct location (\%) } \\
\hline Anterior & 45 & 41 & 39 & 51 & 46 \\
\hline Inferior & 49 & 50 & 61 & 46 & 46 \\
\hline Other & 5 & 9 & 0 & 3 & 8 \\
\hline Previous AMI (\%) & 16 & 21 & 13 & 16 & 14 \\
\hline Previous CABG (\%) & 3 & 3 & 4 & 2 & 4 \\
\hline Time to treatment $(\mathrm{h})$ & $3.1(0.1)$ & $2.9(0.2)$ & $3.2(0.3)$ & $3.2(0.2)$ & $3.1(0.3)$ \\
\hline Time to angiography $(\mathrm{h})$ & $1.6(0.02)$ & $1.6(0.02)$ & $1.6(0.06)$ & $1.6(0.03)$ & $1.6(0.03)$ \\
\hline \multicolumn{6}{|l|}{ Clinical events } \\
\hline Stroke, all types (\%) & 2 & 3 & 0 & 0 & 4 \\
\hline Shock (\%) & 2 & 0 & 4 & 2 & 4 \\
\hline Angioplasty (\%) & 9 & 18 & 17 & 4 & 0 \\
\hline 30 day mortality (\%) & 3 & 3 & 4 & 2 & 3 \\
\hline \multicolumn{6}{|c|}{ TIMI score (number of patients) } \\
\hline 0 & 23 & 14 & 4 & 4 & 1 \\
\hline 1 & 6 & 2 & 4 & 0 & 0 \\
\hline 2 & 36 & 11 & 4 & 12 & 9 \\
\hline 3 & 50 & 5 & 6 & 23 & 16 \\
\hline $\begin{array}{l}\text { Infarct size calculated from } \\
\mathrm{HBDH}(\mathrm{g} \text { eq/l) }\end{array}$ & $4.8(0.3)$ & $5.3(0.5)$ & $5.3(1.0)$ & $4.3(0.5)$ & $4.7(0.8)$ \\
\hline
\end{tabular}

Data are expressed as mean (SEM) or per cent unless specified.

AMI, acute myocardial infarction; CABG, coronary artery bypass graft; iv, intravenous; sc, subcutaneous; SK, streptokinase; TIMI, thrombolysis in myocardial infarction grade; t-PA, accelerated tissue plasminogen activator.

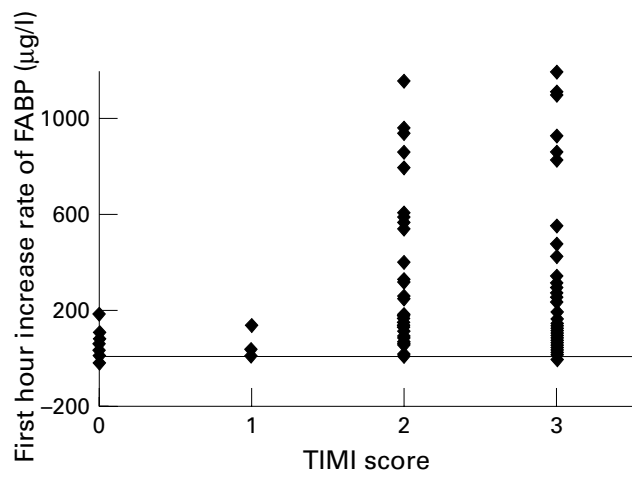

Figure 1 The first hour increase in plasma fatty acid binding protein (FABP) release in patients with TIMI grade $0(n=23)$, TIMI $1(n=6)$, TIMI $2(n=36)$, and TIMI $3(n=50)$. In the TIMI $0+1$, TIMI 2 , and TIMI 3 patients the mean (SEM) increases were 50 (11), 283 (53), and 232 (45) $\mu \mathrm{g} / \mathrm{l} / \mathrm{h}$.

marker the increases were not significantly different between the TIMI 2 and TIMI 3 patients (fig 1 and data not shown).

Figure $2 \mathrm{~A}$ shows the ROC curves of the first hour increase in FABP release when patency was defined as TIMI 3 alone or as TIMI $2+3$, and occlusion was defined as either TIMI $0+1$ or TIMI $0+1+2$. The area under the ROC curve was highest when TIMI $2+3$ values were compared with TIMI $0+1 \quad($ area $=0.760)$, slightly smaller when TIMI 3 was compared with TIMI $0+1$ (area $=0.706, \mathrm{p}<0.05)$, and smallest when TIMI 3 was compared with TIMI $0+1+2($ area $=0.530, \mathrm{p}<0.001)$. For myoglobin similar results were obtained (data not shown). Further analysis was therefore performed on the TIMI $2+3$ patients versus the TIMI $0+1$ patients. The probability of reperfusion (q) on the basis of the first hour increase rate of FABP could be estimated by
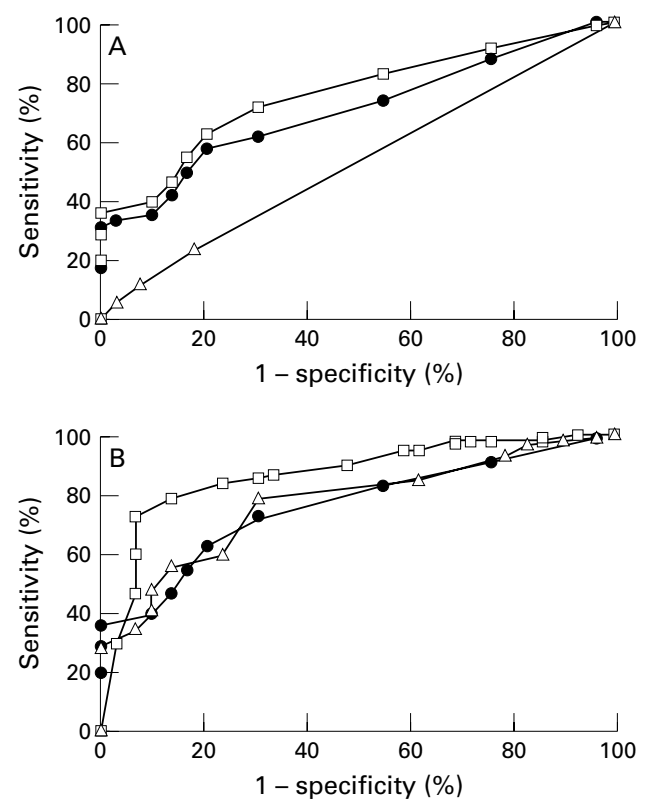

Figure 2 (A) Receiver operating characteristic (ROC) curves (sensitivity $v 1$ - specificity) for the first hour increase of fatty acid binding protein (FABP) release, comparing TIMI grade $3 v$ TIMI $0+1+2$ (empty triangles), TIMI 3 v TIMI $0+1$ (filled circles), and TIMI $2+3 v$ TIMI $0+1$ (empty squares). (B) ROC curves of the first hour increase rate of FABP (filled circles), the first hour increase rate of $F A B P$ and $Q H B D H_{72}$ (empty squares), and the first hour increase rate of FABP plus the sum of ST segment elevations (empty triangles), for TIMI $2+3 v$ TIMI $0+1 . Q H B D H_{72}$, cumulative release of a hydroxybutyrate dehydrogenase into plasma in the first 72 hours.

$\log (\mathrm{q} / 1-\mathrm{q})=0.1367+0.0097 \times$ (first hour increase rate of FABP).

PREDICTION OF REPERFUSION BY FABP, PATIENT CHARACTERISTICS, CLINICAL VARIABLES, AND INFARCT SIZE

We attempted to improve discrimination between reperfused (TIMI 2+3) and nonreperfused patients (TIMI $0+1$ ) by combining the first hour increase in FABP release with the 1-3 hour increase in FABP release, some characteristics of the patients (age, sex), clinical variables (treatment delay, catheterisation delay, infarct location), and infarct size (as measured either by the cumulative release of $\alpha$ hydroxybutyrate dehydrogenase into plasma in the first 72 hours $\left(\mathrm{QHBDH}_{72}\right)$ or by ST segment elevation). Forward stepwise logistic regression analysis indicated that prediction of reperfusion was not improved by adding the 1-3 hour increase in FABP release, the patient characteristics, or the clinical variables to the model ( $p>0.05)$, nor did the addition of infarct size, as measured by ST segment elevation, improve discrimination between reperfused and non-reperfused patients (fig $2 \mathrm{~B}$, area under the ROC curve 0.772). In contrast, the addition of infarct size as measured by $\mathrm{QHBDH}_{72}$ did improve discrimination between reperfused and non-reperfused patients (fig 2B, area under the ROC curve 0.868, $\mathrm{p}<0.001)$.

The probability of reperfusion (q) on basis of the first hour increase in FABP release and enzymatic infarct size could be estimated by: 

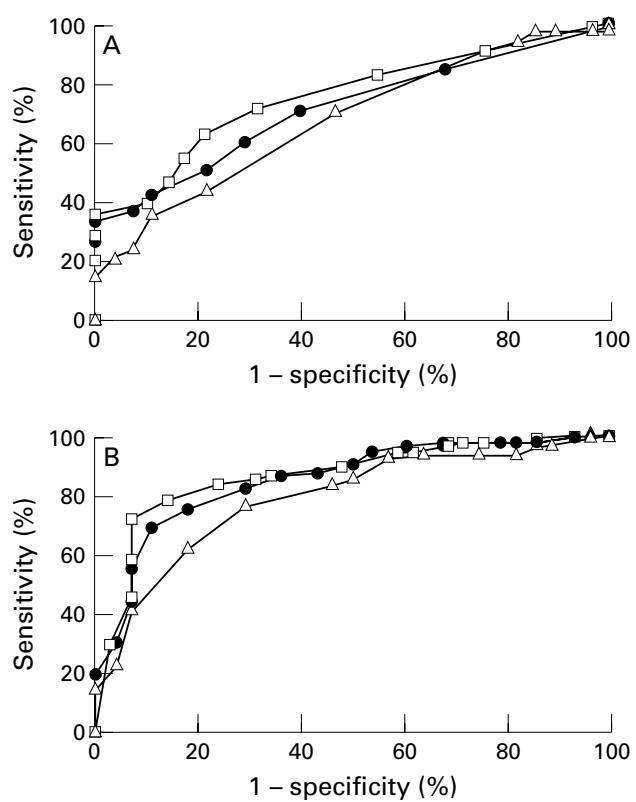

Figure 3 Comparison of fatty acid binding protein (FABP) with myoglobin (MYO) and creatine kinase $M B$ isoenzyme $(C K-M B)$. (A) Receiver operating characteristic (ROC) curves (sensitivity $v 1$ - specificity) of the first hour increase in release of FABP (empty squares), myoglobin (filled circles), or $C K-M B$ (empty triangles), for TIMI grades $2+3$ v TIMI $0+1$. (B) ROC curves combining the first hour increase in release of FABP (empty squares), myoglobin (filled circles), or $C K-M B$ (empty triangles) with $Q H B D H_{72}$, for TIMI $2+3$ v TIMI $0+1$

$\log (\mathrm{q} / 1-\mathrm{q})=1.6542+0.0132 \times$ (first hour increase rate of FABP) $-0.4054 \times$ (enzymatic infarct size). The negative value of $\mathrm{b} 2$ corrects for the extra rapid first hour increase rates in case of larger infarctions.

Table 2 Sensitivity, specificity, positive predictive value (PPV), and negative predictive value (NPV) at optimal points in the receiver operating characteristic (ROC) curve (maximum sum of sensitivity and specificity) for the various reperfusion markers

\begin{tabular}{llllll}
\hline & $\begin{array}{l}\text { Probability of } \\
\text { reperfusion }(q)\end{array}$ & $\begin{array}{l}\text { Sensitivity } \\
(\%)\end{array}$ & $\begin{array}{l}\text { Specificity } \\
(\%)\end{array}$ & $\begin{array}{l}\text { PPV } \\
(\%)\end{array}$ & $\begin{array}{l}\text { NPV } \\
(\%)\end{array}$ \\
\hline $\begin{array}{l}\text { First hour increase rate of: } \\
\text { FABP }(\mu \mathrm{g} / \mathrm{l} / \mathrm{h})\end{array}$ & 0.66 & 71 & 69 & 87 & 44 \\
$\begin{array}{l}\text { Myoglobin }(\mu \mathrm{g} / \mathrm{l} / \mathrm{h}) \\
\text { CK-MB }(\mathrm{U} / \mathrm{l} / \mathrm{h})\end{array}$ & 0.67 & 67 & 71 & 88 & 42 \\
& 0.73 & 52 & 75 & 87 & 34 \\
QHBDH & & & & & \\
FABP $(\mu \mathrm{pg} / \mathrm{l} / \mathrm{h})$ & 0.78 & 74 & 93 & 97 & 55 \\
Myoglobin $(\mu \mathrm{g} / \mathrm{l} / \mathrm{h})$ & 0.78 & 73 & 89 & 95 & 52 \\
CK-MB $(\mathrm{U} / \mathrm{l} / \mathrm{h})$ & 0.75 & 77 & 71 & 89 & 50 \\
\hline
\end{tabular}

$\mathrm{CK}-\mathrm{MB}$, creatine kinase $\mathrm{MB}$ isoenzyme; $\mathrm{FABP}$, fatty acid binding protein; $\mathrm{QHBDH}_{72}$, cumulative release of $\alpha$ hydroxybutyrate dehydrogenase in the first 72 hours.

Table 3 Sensitivity, specificity, positive predictive value (PPV), and negative predictive value (NPV) for reperfusion, using the composite variable $q$ (probability of reperfusion)

\begin{tabular}{lllll}
\hline Probability of reperfusion $(q)$ & Sensitivity (\%) & Specificity (\%) & PPV (\%) & NPV (\%) \\
\hline 0.20 & 98 & 14 & 77 & 67 \\
0.40 & 97 & 31 & 81 & 75 \\
0.55 & 90 & 52 & 85 & 63 \\
0.60 & 87 & 66 & 88 & 63 \\
0.77 & 76 & 90 & 96 & 55 \\
0.80 & 73 & 93 & 97 & 54 \\
0.95 & 30 & 97 & 97 & 32 \\
\hline
\end{tabular}

Values for q were calculated from the first hour increase rate of plasma fatty acid binding protein (FABP) and enzymatic infarct size according to the equation:

$\log (q / 1-q)=1.6542+0.0132 \times($ first hour increase rate of FABP $)-0.4054 \times\left(\mathrm{QHBDH}_{72}\right)$, where $\mathrm{QHBDH}_{72}=$ cumulative release of $\alpha$ hydroxybutyrate dehydrogenase in the first 72 hours.
COMPARISON OF FABP, MYOGLOBIN, AND CK-MB AS REPERFUSION MARKERS

As with FABP, the reperfusion predicting model was improved when the first hour increase in myoglobin release was combined with enzymatic infarct size (the values of b0, b1, and b2 were $1.6839,0.0034$, and -0.3905 , respectively, while ROC curve areas for myoglobin alone and for myoglobin in combination with enzymatic infarct size were 0.718 and $0.857, \mathrm{p}=0.004$, fig $3 \mathrm{~A}$ and $3 \mathrm{~B}$ ). The area under the ROC curve of the first hour increase rate of $\mathrm{CK}-\mathrm{MB}$ release was also augmented by adding enzymatic infarct size to the model (0.681 and 0.769 , respectively, $\mathrm{p}=0.04$; values of b0, b1, and b2 were 1.9102, 0.0736, and -0.2402 , respectively.). The areas under the ROC curve for the first hour increase in FABP and myoglobin release combined with enzymatic infarct size were both larger than for CK-MB ( $p=0.046$ and $p=0.028$, respectively) (fig $3 \mathrm{~A}$ and $3 \mathrm{~B}$ ).

Using the ROC curves, optimum points (giving the maximum sum of sensitivity and specificity) of the first hour increase in release of FABP, myoglobin, and CK-MB were determined. In addition to sensitivity and specificity, the positive and negative predictive values were calculated at these points (table 2). Various probabilities of reperfusion predicted by FABP are also shown in combination with these variables (table 3).

\section{Discussion}

In this multicentre study, FABP was used as a marker to distinguish successful reperfusion from persistent occlusion after thrombolytic treatment in a large group of patients with confirmed acute myocardial infarction. The definition of reperfusion was either TIMI $2+3$ score or TIMI 3 score alone. Our findings indicate that the best discrimination between reperfusion and occlusion by plasma FABP or myoglobin was obtained when TIMI $2+3$ were classified as reperfusion and TIMI $0+1$ as occlusion. FABP was found to perform as well as plasma myoglobin, while both variables performed better than CK-MB. For acute clinical decision making, the accuracy of the first hour increase in FABP release appeared too low. However, in retrospective studies it may be useful to include both the first hour increase in FABP (or myoglobin) release and the enzymatic infarct size in the predictive model.

\section{FIRST HOUR INCREASE RATE OF FABP RELEASE} AFTER THROMBOLYSIS AS A REPERFUSION MARKER FABP is a small cytoplasmic protein $(15 \mathrm{kDa})$, like myoglobin $(17.8 \mathrm{kDa})$, which is rapidly released from injured myocardium and enters the bloodstream when the occluded artery is reopened by thrombolytic agents. ${ }^{2122}$ Peak values are reached on average within six hours after the onset of acute myocardial infarction and return to normal within 24 hours. $^{82122}$ Earlier studies used the time to the peak plasma concentration to assess the value of serum markers for discriminating between reperfused and non-reperfused patients. For myoglobin, 
Zabel and colleagues showed that measurement of the early initial slope gives a more accurate assessment of reperfusion than the time to peak concentration, and it is also more rapidly available. ${ }^{6}$ The first hour increase in release can be assessed either as an absolute value (value at time $\mathrm{t}$ - value at time $0 / \mathrm{dt}$ ) or relatively (value at time $t /$ value at time 0 ). In an ROC curve analysis for myoglobin, Tanasijevic and colleagues found no differences in discrimination when comparing absolute or relative early slope values. ${ }^{23}$ In the present study the absolute first hour increase in FABP release was assessed, and the individual data showed great variation and considerable overlap in the first hour release values among the various TIMI scores.

DEFINITION OF REPERFUSION

In many studies, TIMI 2 and 3 patients have been grouped and classified as being reperfused. ${ }^{672123-27}$ However, a study by Anderson and colleagues showed that TIMI grade 3 blood flow resulted in improved patient outcome compared with grade 2 flow. ${ }^{28}$ For this reason, the TIMI 3 score alone may be defined as successful reperfusion, while the TIMI 2 score represents an intermediate point between TIMI 3 and TIMI $0+1$. In the present study we considered both TIMI 3 only and TIMI $2+3$ combined as reperfusion. The results for TIMI $3 v$ TIMI $0+1$ or $v 0+1+2$ were less satisfactory, while the best performance with FABP was obtained when both TIMI 2 and 3 groups were considered as being reperfused. The results were similar for myoglobin and CK-MB (data not shown). Hence FABP may be useful as a reperfusion marker in discriminating between TIMI $2+3 v$ TIMI $0+1$, but not in differentiating between TIMI 2 or TIMI 3. Similarly, Christenson and colleagues reported a better performance for myoglobin when TIMI $2+3$ were compared with TIMI $0+1$, rather than when comparing TIMI 3 with TIMI $0+1+2 .{ }^{27}$ Moreover, Apple recently concluded that TIMI 3 flow patients cannot be differentiated from TIMI 2 patients by using biochemical markers. ${ }^{29}$

INCLUDING INFARCT SIZE IN THE MODEL

When the amount of necrosis is small, the first hour increase in release of cell components may be low and values found in the reperfused group may overlap with those seen in patients without reperfusion but with larger infarcts. This overlap can be reduced when rates of release are combined with enzymatic infarct size in the model. However, a drawback of using the cumulative release of $\alpha$ hydroxybutyrate dehydrogenase over 72 hours as a measure of infarct size is the long time necessary for sample collection; hence this method is only useful in retrospective studies. Because of this, we also evaluated the use of the sum of the initial ST elevations as a surrogate for infarct size. A very weak correlation was found between the sum of ST elevations and the cumulative release of $\alpha$ hydroxybutyrate dehydrogenase over 72 hours $(r=0.36$, fig 4$)$. Similarly, Willems and colleagues also showed a poor correlation between the sum of the initial ST

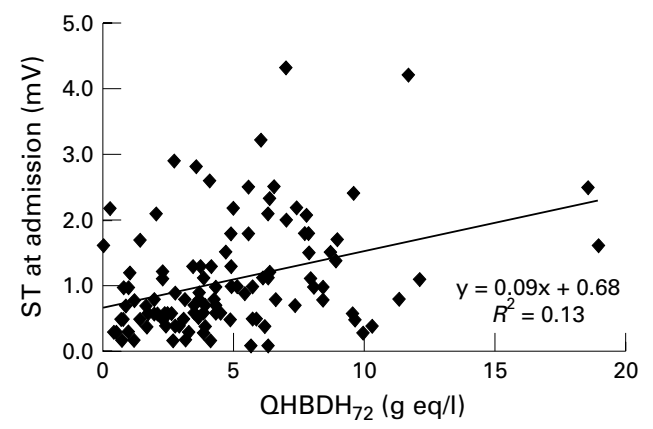

Figure 4 Scatterplots of infarct size estimated from the sum of ST segment elevations in the admission ECG and from cumulative release of a hydroxybutyrate dehydrogenase during 72 hours $\left(Q H B D H_{72}\right)$. The best fit straight line (drawn) is shown.

elevations and enzymatic infarct size $(r=0.32){ }^{30}$ On the other hand, Yusuf and associates found a much higher correlation $(r=0.73)$, but they used a more refined technique (precordial mapping) to evaluate ST deviations. ${ }^{31}$ Using the sum of ST segment elevation in our study, no improvement of discrimination between reperfused and nonreperfused patients could be obtained (fig $2 \mathrm{~B}$ ).

COMPARISON OF FABP, MYOGLOBIN, AND CK-MB AS REPERFUSION MARKERS

Various biochemical markers of myocardial necrosis have been used to detect reperfusion non-invasively after thrombolysis. These include measurement of myoglobin and CK$\mathrm{MB}^{5}{ }^{525-27}$ In the present study, we compared FABP with myoglobin and CK-MB as reperfusion marker by ROC curve analysis. Similar results were found for FABP and myoglobin, while CK-MB was slightly worse as a reperfusion marker. These findings are in line with earlier studies in indicating more accurate detection of reperfusion by myoglobin than by $\mathrm{CK}-\mathrm{MB},{ }^{6}{ }^{24} 25$ while in a very recent study by Ishii and colleagues on 45 patients, comparable results were obtained for FABP and myoglobin. ${ }^{21} \mathrm{FABP}$ is relatively more cardiospecific than myoglobin as the (heart type) FABP content of skeletal muscle is considerably less than in cardiac muscle, while the muscular content of myoglobin is approximately twice that of the heart (when expressed per $g$ wet weight of muscle). ${ }^{22}$ In case of muscle injuryfor example, electrical defibrillation, intramuscular injection, or trauma-the serum concentration of myoglobin will rise rapidly and hence false positive results could be obtained. However, in the present work, as well as in that of Ishii and colleagues, patients with these conditions were excluded. ${ }^{21}$

The sensitivity and specificity of FABP, myoglobin, and CK-MB, calculated at the optimum points of the ROC curve, were not as high in the present study as in others (table 4). This may reflect the fact that in the present study blood sampling was not accompanied by repeated angiography and so the exact timing of the reopening of the vessel was not known. Increased rates of cell component release were determined during the first hour after thrombolytic treatment, while the angiogram was taken 
Table 4 Sensitivity and specificity of fatty acid binding protein (FABP), myoglobin, and creatine kinase MB isoenzyme (CK-MB) in detection of coronary reperfusion within 60 minutes of the start of treatment: a comparison of several studies

\begin{tabular}{|c|c|c|c|c|c|c|c|c|}
\hline First author, reference & $\begin{array}{l}\text { Number of } \\
\text { patients }\end{array}$ & $\begin{array}{l}\text { Reperfused } \\
\text { patients (\%) }\end{array}$ & $\begin{array}{l}\text { FABP sensitivity } \\
(\%)\end{array}$ & $\begin{array}{l}\text { FABP } \\
\text { specificity (\%) }\end{array}$ & $\begin{array}{l}\text { Myoglobin } \\
\text { sensitivity (\%) }\end{array}$ & $\begin{array}{l}\text { Myoglobin } \\
\text { specificity (\%) }\end{array}$ & $\begin{array}{l}C K-M B \\
\text { sensitivity (\%) }\end{array}$ & $\begin{array}{l}C K-M B \\
\text { specificity (\%) }\end{array}$ \\
\hline$\overline{\mathrm{Abe}^{7}}$ & 31 & 65 & - & - & 95 & 100 & - & - \\
\hline Ishii $^{25}$ & 49 & 65 & - & - & 97 & 100 & 84 & 100 \\
\hline Ishii $^{21}$ & 45 & 67 & 97 & 100 & 93 & 100 & - & - \\
\hline Miyata $^{24}$ & 63 & 71 & - & - & 100 & 89 & 91 & 67 \\
\hline Ohman $^{26}$ & 207 & 71 & _- & _- & - & - & 78 & 67 \\
\hline Tanasijevic $^{23}$ & 27 & 70 & - & - & 89 & 100 & 83 & 100 \\
\hline $\mathrm{Zabel}^{6 \star}$ & 63 & 73 & - & - & 94 & 88 & 85 & 71 \\
\hline \multicolumn{9}{|l|}{ Present study: } \\
\hline - Increase rate FABP & 115 & 75 & 71 & 69 & 67 & 71 & 52 & 75 \\
\hline $\begin{array}{l}\text { - Increase rate FABP and } \\
\mathrm{QHBDH}_{72}\end{array}$ & 115 & 75 & 74 & 93 & 73 & 89 & 77 & 71 \\
\hline
\end{tabular}

^Blood sampling 90 minutes after treatment.

later (between 0.9 and 2.0 hours after treatment). A discrepancy between protein indices and angiographic assessment of reperfusion may have occurred in patients with late reperfusion, early spontaneous reperfusion, or reocclusion of the infarct related artery after initial successful thrombolysis. In an attempt to account for late reperfusion, we added the increase rate of FABP (per hour) at 1-3 hours after thrombolytic treatment to our model, but no additional discrimination was obtained. However, the possibility of early spontaneous reperfusion or reocclusion after initial successful thrombolysis could not be excluded, because a pretreatment coronary angiogram was missing and because of insufficient blood sampling. Notwithstanding these considerations, our data still allow an adequate comparison of the performance of the three plasma markers studied.

CLINICAL APPLICATIONS

In the absence of angiography, it is important that the FABP test can identify patients with persistent occlusion, because such patients can be treated by other interventions such as rescue percutaneous transluminal coronary angioplasty. Thus both the negative predictive value and the specificity were considered. At the optimal point in the curve, the negative predictive value-indicating the percentage of correctly identified patients with decreased rates of release-was low (table 2 ). The negative predictive value is related to the prevalence of non-reperfused patients, which was relatively low $(25 \%)$ in the present study. The optimum specificity of the first hour increase in release of FABP was $69 \%$. In practice, this would mean that on the basis of the first hour increase in FABP release, $31 \%$ of the patients with TIMI 0 or 1 score would not be treated further after failing thrombolytic treatment-a rather high percentage. When the specificity increases - that is, fewer patients with closed infarct related arteries are missed-the sensitivity simultaneously decreases (more patients with adequate perfusion after thrombolytic treatment will be treated unnecessarily). It depends on the clinical management which factor (sensitivity or specificity) is regarded as the most important. By using two probability values and disregarding the patients who fell in between, a population of patients with acute myocardial infarction treated with thrombolytic agents and without coronary angiography could be divided into a reperfused group and a non-reperfused group. Table 3 shows that in this way a group of patients with a $>97 \%$ chance of successful reperfusion and a group with a $>75 \%$ chance of non-reperfusion could be obtained by retrospective study. The latter figure of $75 \%$ is relatively low because of the low first hour increase in rate of FABP in reperfused patients with small infarcts. This figure would thus have been higher if our analyses had been restricted to patients with a certain minimum infarct size (for example, $1 \mathrm{~g}$ eq/1).

The performance of FABP as a reperfusion marker is greatly improved by including enzymatic infarct size in the model. For example, a patient with an average first hour increase of plasma FABP of $200 \mu \mathrm{g} / \mathrm{l} / \mathrm{h}$ and a large infarct ( $>10 \mathrm{~g}$ eq/1) — according to the formula presented in the legend to table 3-would only have $a<63 \%$ chance (that is, $q=0.63$ ) of successful reperfusion, whereas if this patient had a small infarct $(<1 \mathrm{geq} / \mathrm{l})$ the chance of successful reperfusion would be $>99 \%$ (that is, $q=0.99)$.

\section{CONCLUSIONS}

As a non-invasive reperfusion marker, FABP performs as well as myoglobin. Our data indicate that for acute clinical decision making there is inadequate discrimination between reperfusion and non-reperfusion using this method, but in retrospective studies discrimination can be improved by considering both the increase rate of plasma FABP and the enzymatically determined infarct size.

We gratefully acknowledge the expert technical assistance of Y F de Jong, M M A L Pelsers, M A J G Fischer, M L Mullers-Boumans, and B Kop-Klaassen. The study was supported by the Netherlands Heart Foundation grant 95.189.

\footnotetext{
1 Kircher BJ, Topol EJ, O'Neill Ww, et al. Prediction of infarct coronary artery recanalization after intravenous thrombo-

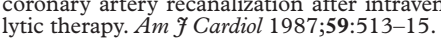

2 Califf RM, O'Neill W, Stack RS, et al. Failure of simple clinical measurements to predict perfusion status after intracal measurements to predict perfusion status after in

venous thrombolysis. Ann Intern Med 1988;108:658-62.
3 Ellis AK, Little T, Masud ARZ, et al. Early noninvasive detection of successful reperfusion in patients with acute myocardial infarction. Circulation 1988;78:1352-7.

4 Lewis BS, Ganz W, Laramee P, et al. Usefulness of a rapid initial increase in plasma creatine kinase activity as a marker of reperfusion during thrombolytic therapy for acute myocardial infarction. Am $\mathcal{f}$ Cardiol 1988;62:20-4.

5 Garabedian HD, Gold HK, Yasuda T, et al. Detection of coronary artery reperfusion with creatine kinase-MB determinations during thrombolytic therapy: correlation with acute angiography. $\mathcal{F} \mathrm{Am}$ Coll Cardiol 1988;11:729-34.

6 Zabel M, Hohnloser SH, Köster W, et al. Analysis of creatine kinase, CK-MB, myoglobin, and troponin T timeine kinase, CK-MB, myoglobin, and troponin T timeactivity curves for early assessment of coronary artery reperfusion after intravenous thrombolysis. Circulation

7 Abe S, Arima S, Nomoto K, et al. Early detection of coronary reperfusion by rapid assessment of plasma myoglobin.
Int $\mathcal{F}$ Cardiol 1993;38:33-40.
} 
8 Glatz JFC, Kleine AH, Van Nieuwenhoven FA, et al. Fattyacid-binding protein as a plasma marker for the estimation of myocardial infarct size in humans. Br Heart $f$ 1994;71:135-40

9 Tanaka T, Hirota Y, Sohmiya K, et al. Serum and urinary human heart fatty acid-binding protein in acute myocardial infarction. Clin Biochem 1991;24:195-201.

10 Glatz JFC, Haastrup B, Hermens WT, et al. Fatty acid binding protein and the early detection of acute myocardial infarction: the EUROCARDI multicenter trial [abstract] Circulation 1997;96(suppl 1):I-215.

11 Ishii J, Wang J, Naruse $\mathrm{H}$, et al. Serum concentrations of myoglobin vs human heart-type cytoplasmic fatty acidbinding protein in early detection of acute myocardial infarction. Clin Chem 1997; 43:1372-8.

12 Willems GM, Visser MP, Krill MTA, et al. Quantitative analysis of plasma enzyme levels based upon simultaneous determination of different enzymes. Cardiovasc Res 1982; 16:120-31.

13 Baardman T, Hermens WT, Lenderick T, et al. Differential effects of tissue plasminogen activator and streptokinase on infarct size and on rate of enzyme release: influence of early infarct related artery patency. The GUSTO Enzyme Substudy. Eur Heart f 1996;17:237-46.

14 The GUSTO Angiographic Investigators. The effects of tissue plasminogen activator, streptokinase, or both on coronary-artery patency, ventricular function, and survival after acute myocardial infarction. $N$ Engl F Med 1993;329: 1615-22.

15 The TIMI study group. The thrombolysis in myocardial infarction (TIMI) trial. Phase I findings. $N$ Engl f Med 1985;312:932-6.

16 Wodzig KWH, Pelsers MMAL, Van der Vusse GJ, et al. One-step enzyme-linked immunosorbent assay (ELISA) for plasma fatty acid-binding protein. Ann Clin Biochem 1997;34:263-8

17 De Groot MJM, Wodzig KWH, Simoons ML, et al. Measurement of myocardial infarct size from plasma fatty acid-binding protein or myoglobin, using individually estiacid-binding protein or myoglobin, using individually

18 Bär FW, Vermeer F, De Zwaan C, et al. Value of admission electrocardiogram in predicting outcome of thrombolytic therapy in acute myocardial infarction. Am f Cardiol 1987; 59:6-13.

19 Fienberg SE. The analysis of cross-classified categorical data. London: MIT Press, 1980.
20 Hanley JA, McNeil BJ. A method of comparing the areas under receiver operating characteristic curves derived from 983:148:839-43.

21 Ishii J, Nagamura Y, Nomura M, et al. Early detection of successful coronary reperfusion based on serum concentration of human heart-type cytoplasmic fatty acid-binding protein. Clin Chim Acta 1997;262:13-27.

22 Van Nieuwenhoven FA, Kleine AH, Wodzig KWH, et al. Discrimination between myocardial and skeletal muscle injury by assessment of the plasma ratio of myoglobin over fatty acid-binding protein. Circulation 1995;92:2848-54

23 Tanasijevic M, Cannon CP, Wybenga DR, et al. Myoglobin, creatine kinase $M B$, and cardiac troponin-I to assess reperfusion after thrombolysis for acute myocardial infarction: results from TIMI 10A. Am Heart f 1997;134:622-30.

24 Miyata M, Abe S, Arima S, et al. Rapid diagnosis of coronary reperfusion by measurement of myoglobin level every $15 \mathrm{~min}$ in acute myocardial infarction. $\mathcal{F} \mathrm{Am}$ Coll Cardiol 1994:23:1009-15.

25 Ishii J, Nomura M, Ando T, et al. Early detection of successful coronary reperfusion based on serum myoglobin ful coronary reperfusion based on serum myoglobin concentration: comparison with serum creatine kina
isoenzyme MB activity. Am Heart f 1994;128:641-8.

26 Ohman EM, Christenson RH, Califf RM, et al. Noninvasive detection of reperfusion after thrombolysis based on serum creatine kinase $\mathrm{MB}$ changes and clinical variables. Am Heart f 1993;126:819-26.

27 Christenson RH, Ohman EM, Topol EJ, et al. Assessment of coronary reperfusion after thrombolysis with a model combining myoglobin, creatine kinase-MB, and clinical variables. Circulation 1997;96:1776-82.

28 Anderson JL, Karagounis LA, Becker LC, et al. TIMI perfusion grade 3 but not grade 2 results in improved outcome after thrombolysis for myocardial infarction: ventriculographic, enzymatic, and electrocardiographic evidence from the TEAM-3 study. Circulation 1993;87:182939.

29 Apple FS. Biochemical markers of thrombolytic success. Scand $\mathcal{F}$ Clin Lab Invest 1999;59(suppl 230):60-6.

30 Willems JL, Willems RJ, Willems GM, et al. Significance of initial ST segment elevation and depression for the management of thrombolytic therapy in acute myocardial infarction. Circulation 1990;82:1147-58.

31 Yusuf S, Lopez R, Maddison A, et al. Value of electrocardiogram in predicting and estimating infarct size in man. $\mathrm{Br}$ Heart f 1979;42:286-93.

\section{IMAGES IN CARDIOLOGY}

\section{Pneumopyopericardium, complicating penetrating gastric ulcer}

A 66 year old man was admitted because of monosymptomatic fever. Acute laboratory findings indicated severe infection and ECG showed low voltage and atrial fibrillation with a heart rate of 116 beats $/ \mathrm{min}$. A chest radiograph (right) showed pneumopericardium, making transthoracic echocardiography (TTE) impossible. Transoesophageal echocardiography (TOE), however, disclosed normal heart function but echodense material indicating pus in the pericardium behind the heart. Surgery and endoscopy showed pneumopyopericardium caused by a gastric ulcer, penetrating through the diaphragm to the pericardium. The patient was treated successfully with pericardial drainage, prolonged antibiotic therapy, and surgical resection of the gastropericardial fistula. Gastrointestinal fistula's are well established causes of pneumo- and pyopericardium.

Chest radiography is an excellent imaging technique for establishing the diagnosis of pneumopericardium but is of limited value in the analysis of a potential cardiac component

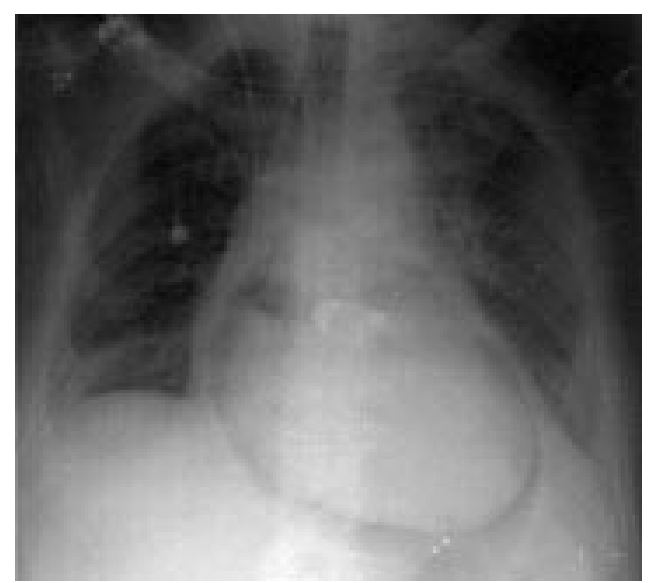

of shock. In such cases, where air obstructs the window of TTE, TOE may be useful.

ANNE SEJR KNUDSEN SVEND EGGERT JENSEN HENRIK EGEBLAD asknudsen@dadlnet.dk 